\title{
Is it Rational to Minimize Tax Payments?
}

\author{
Andreas Löffler, Lutz Kruschwitz \\ Universität Paderborn, Lehrstuhl für Finanzierung und Investition, Paderborn, Germany \\ Freie Universität Berlin, Institut für Bank-und Finanzwirtschaft, Berlin, Germany \\ E-mail:AL@wacc.de,LK@wacc.de \\ Received March 15, 2010; revised April 5, 2010; accepted April 25, 2010
}

\begin{abstract}
The opinion is occasionally voiced that investors should avoid paying tax at all costs. In this paper it is being investigated, using a simple portfolio model with taxes, whether avoiding tax really leads to more $\mu$ - $\sigma$-efficient solutions. It is demonstrated for four different concepts of tax-minimising policy that they are a far cry from an efficient solution.
\end{abstract}

Keywords: Decision, Taxes, CAPM, Tax-CAPM

\section{Problem Outline}

Considering the question on how to increase one's net income, an investor has two options. He or she will either look for opportunities to increase the pre-tax income, or alternatively to diminish tax payments. Many people are assumed to especially favor tax saving schemes. A few years ago, a German economist, Ekkehard Wenger, has written on this predilection, which he considers thoroughly reasonable. Rationally acting investors would either make legal use of tax incentives in their investments, or even attempt tax evasion. Anyone acting differently would be "maximizing stupidity" [1]. Regardless of whether one agrees or not with such drastic views, the rationale behind such a position seems to make sense. As a scientist however, one should be armed with a fundamentally skeptical outlook; easily comprehended matters do not always fit in with reality, as one readily learns in school from the conundrum involving Achilles and the turtle.

Wenger's considerations are relevant in view of empirical analyses of the stock market. Fair evaluations of companies' values are normally performed by using Capital Asset Pricing Models (CAPM). This involves the comparison with similar companies, in order to determine the so-called "beta factor" of the firm in question. Particularly the financial auditor profession is adamant that for this determination not the basic model of CAPM be used, but rather its tax-enabled version, the TaxCAPM [2]. The values resulting from the respective uses of CAPM and Tax-CAPM can sometimes vary significantly. If one subscribes to the opinion that tax payments reflect irrational behavior, it follows that Tax-CAPM will yield incorrect company valuations. Thus, the question of whether saving taxes constitutes rational behavior, directly affects what a company is worth.

In our present contribution we will, while using the framework of portfolio theory, examine whether a policy of minimizing taxes can be regarded as advantageous. For this purpose, we consider a simple portfolio problem from a tax perspective and attempt a characterization of all portfolios which are efficient under uncertainty. Such portfolios can be characterized as desirable even without more precise knowledge on the risk averseness of investors. On this basis we will examine the question, how the concept of "tax minimization" can be formalized in our context and if such concepts can be efficient. It will emerge that tax minimizers, while maybe considering themselves to be particularly clever, end up looking less than smart.

We will concentrate our analysis exclusively on portfolio theory and will not employ the CAPM. This procedure follows from the goal we have set for ourselves. We would like to show that even rational investors can be willing payers of taxes, as they prefer a balanced aftertax structure of payments. What kind of macroeconomic result would be the upshot of such behavior is outside the scope of our work.

\section{The Model}

We use a formal model which is based on definitions and simplifying assumptions deemed appropriate. On this basis we will use logical operations to draw conclusions whose validity can be checked by an expert third person at any time. Given that we do not make mistakes during 
the logical operations, our results can only be legitimately criticised by referring to a possible use of inappropriate assumptions.

\subsection{Assumptions}

We are looking at a one period model under uncertainty. An investor has the option to invest in risky securities $j=1, \ldots, J$. The price vector of these investments is called $p=\left(p_{1}, \ldots, p_{J}\right)$. We notate future payments connected with the securities with $\tilde{Y}_{j}$. We use the symbol $E=\left(E\left[\tilde{Y}_{1}\right], \ldots, E\left[\tilde{Y}_{J}\right]\right)$ for the vector of the expected cashflows. The covariance matrix of the cashflows is $\Omega$. It is assumed that the covariance matrix is regular. Therefore there are no redundant investments. A risk free asset will not be introduced. But we concentrate on the edge of an "eggshell-like surface" which may be interpreted as the geometric place of all desirable risk-return-positions and uses to be called the efficient set.

We imply an investor with nominal assets of $\omega$, who has already financed his momentary consumption and has to decide how to invest the amount $\omega$ in the capital market. For that purpose he chooses a portfolio consisting of $N=\left(N_{1}, \ldots, N_{J}\right)$ units of the risky assets. Short selling is not excluded. Therefore some entries in this vector may also be negative. When deciding on his investment the investor orients himself by the expectation value and variance. He thus has a utility function of the type

$$
U\left(\mu, \sigma^{2}\right)
$$

According to our requirements the expectation value and variance of the future payments of a securities portfolio are determined to $\mu=N \cdot E$ and $\sigma^{2}=N \cdot \Omega \cdot N$. The price of the portfolio is given by the vector product $N \cdot p$.

In order to model the taxation an assessment basis as well as a tax-rate function is required. As assessment basis for a unit of the $j$-th financial asset we us the difference between the risky cashflow and a riskless depreciation which in the simplest case corresponds to the purchase price, but not necessarily. We term the vector of the depreciations of the risky assets as $A=\left(A_{1}, \ldots, A_{J}\right)$. The rate function is linear, the tax rate $\tau$ is secure.

\subsection{Efficient Portfolios before and after Taxes}

First we look at the decision under the simplifying assumption that no taxes are being levied. Then we deal with the classic problem of portfolio selection, introduced into the literature by Markowitz more than 50 years ago [3]. The investor can choose between positions charted on an $\mu-\sigma^{2}$-diagram laid out on an eggshell-shaped surface. The most interesting points on this surface are those around the edge, as it is there that for any given expected wealth the variance will be minimal. All positions which are not situated on the edge are denoted as non-efficient. Any rational investor will attempt to occupy an $\mu-\sigma^{2}$ efficient position. In order to determine these edge positions, a maximization problem

$$
\max _{N} N \cdot E
$$

under budget constraints

$$
N \cdot p=\omega \text { and } N \cdot \Omega \cdot N=\sigma^{2}
$$

has to be solved. The first condition is a budget restriction which ensures that the investor's wealth is fully exhausted; the second condition makes sure that the cash flow variance of a portfolio will reach an exogenously demanded level $\sigma^{2}$. In order to fully determine the efficient set, the optimization problem has to be solved for all possible $\sigma^{2} \in \mathbb{R}^{+}{ }^{1}$ Because short sales are not excluded, the optimization can be accomplished by using a Lagrange function. The specific solution is irrelevant to this discussion.

We now focus our attention on after-tax-efficient portfolios. Here, when dealing with cash flows, we must note that taxes are due. An investor holding one unit of asset $j$ will have to deliver the amount $\tau\left(\tilde{Y}_{j}-A_{j}\right)$ to the tax authority. Efficient portfolios are those, which for any given variance and nominal wealth $\omega$, will maximize the expected value $e$. The expected cash flows after taxes then amount to $E-\tau(E-A)=E(1-\tau)+\tau A$. The pre-tax covariance matrix $\Omega$ changes to the post-tax covariance matrix

$$
\begin{aligned}
& \operatorname{Cov}\left[\tilde{Y}_{j}(1-\tau)+\tau A_{j}, \tilde{Y}_{k}(1-\tau)+\tau A_{k}\right] \\
& =(1-\tau)^{2} \operatorname{Cov}\left[\tilde{Y}_{j}, \tilde{Y}_{k}\right]
\end{aligned}
$$

Thus, we now have to maximize the function $N \cdot(E(1-\tau)+\tau A) \quad$ under the budget constraints $N \cdot p=\omega$ and $N \cdot(1-\tau)^{2} \Omega \cdot N=\sigma^{2}$. So long as the amount of write-offs $A$ is not specified, there are many solutions to this problem. However, in a one-period model, $A=p$ represents a plausible-even naturalchoice. The function to be maximized now takes the

${ }^{1}$ If cash flows are not perfectly negatively correlated there are no portfolios having zero variance. Therefore, there is a minimum variance that cannot be undercut. 
form $N \cdot E(1-\tau)+\tau \omega$. Since a positive linear transformation will affect the target function but not the solution itself, we can similarly denote the optimization problem after taxes in the form

$$
\max _{N} N \cdot E
$$

under auxiliary conditions

$$
N \cdot p=\omega \text { and } N \cdot \Omega \cdot N=\frac{\sigma^{2}}{(1-\tau)^{2}}
$$

We recognize at once: The maximization problem after taxes differs from that before taxes in only one, and furthermore irrelevant, respect. In the second budget constraint, $\sigma^{2}$ became the parameter $\frac{\sigma^{2}}{(1-\tau)^{2}}$. It follows that the set of all parameters for both maximization problems must be identical. The efficient set before taxes coincides with the efficient set after taxes.

\subsection{Tax-Minimizing Portfolios}

We begin by stating that, in the context of our discussion, it is not immediately clear what tax avoidance or tax minimization mean. We see several possible ways to specify the concept of tax minimization, if we are dealing with identifying a risky portfolio. Four specific alternatives shall be considered. In each case, we will assume that the risky projects will be completely written off, thus $A=p$.

\subsubsection{Minimizing Taxes in the Worst-Case Scenario}

To formalize this concept of tax minimization, we assume that the number of relevant states at time $t=1$ is finite and that the state-dependent cash flows of assets can be characterized by $Y(s), s=1, \ldots, S$. Then $\tau N \cdot(Y(s)-p)=\tau N \cdot Y(s)-\tau \omega$ describes the tax payments coming due for an investor if the state $s$ occurs in $t=1$. In the worst case, this payment will amount to $\max _{s} \tau N \cdot Y(s)-\tau \omega$. Our first version of tax minimization may amount to choosing portfolio $N$ in such a manner, as to minimize the highest tax amount possible

$$
\min _{N} \max _{s} \tau N \cdot Y(s)
$$

under auxiliary constraint $N \cdot p=\omega$. The solution can be found by means of an appropriate algorithm of linear programming. It is obvious that such a solution is not $\mu-\sigma^{2}$-efficient.

\subsubsection{Absolute Minimization of Expected Taxes}

The expected tax payments of portfolio $N$ amount to $\tau N \cdot(E-p)$. Due to the budget restriction $N \cdot p=\omega$, the optimization problem reads

$$
\min _{N} \tau(N \cdot E-\omega),
$$

if no additional auxiliary conditions are taken into account. The solution is not lower-bound. The expected tax payment tends towards infinite minus. This result does not make economic sense.

\subsubsection{Fading Expected Taxes}

In order to avoid the just mentioned outcome, we can restrict the search for a portfolio, for which the expected tax payments disappear, i.e.,

$$
\tau(N \cdot E-\omega) \stackrel{!}{=} 0 .
$$

For a positive tax rate, the expected tax payment will tend towards zero if the basis of assessment disappears. This is the case, if the expected payments from the portfolio are equal to the invested capital, a slightly surprising, but equally disappointing outcome. Without earning no taxes are due. But would someone cancel one's net earnings in order to save taxes? This would be as unreasonable as if a firm maximized its wages in order to minimize corporate taxes.

2.3.4. Minimizing Expected Taxes on a Given Variance As a final version of a tax-minimizing policy, we consider

$$
\min _{N} \tau(N \cdot E-\omega)
$$

under the constraints

$$
N \cdot p=\omega \text { and } N \cdot \Omega \cdot N=\frac{\sigma^{2}}{(1-\tau)^{2}}
$$

this version of tax minimization we do not have any economics-driven intuition, excluding maybe that the determination of an efficient portfolio without taking variance into account does not seem possible. As the constants in the target function can be ignored, we can rewrite it in the form $\min _{N} N \cdot E$. Comparing this optimization problem with the procedure involving Equations (1) and (2), it becomes immediately clear, that no $\mu-\sigma^{2}$-efficient portfolios can be determined in this manner. A rational investor does not minimize the expected taxes at a given variance. He or she will instead maximize them.

\section{Conclusions}

In the framework of a simple portfolio model with taxes it was considered whether the strategy of tax avoidance will lead to $\mu-\sigma^{2}$-efficient solutions. The examination 
of four distinct concepts of tax minimization strategy has proven that each of them (by far) misses an efficient solution. Thus, when evaluating companies, one must avoid the classic CAPM for determining beta factors and should instead employ a Tax-CAPM.

\section{References}

[1] E. Wenger, "Verzinsungsparameter in der Unternehmensbewertung: Betrachtungen aus theoretischer und em- pirischer Sicht," in German, Die Aktiengesellschaft, Vol. 50, 2005, pp. 9-22.

[2] M. Jonas, A. Löffler and J. Wiese, "Das CAPM mit deutscher Einkommensteuer," in German, Die Wirtschaftsprüfung, Vol. 57, No. 17, 2004, pp. 898-906.

[3] H. M. Markowitz, "Portfolio Selection," The Journal of Finance, Vol. 7, No. 1, 1952, pp. 77-91. 